\title{
Fake News als soziolinguistisches Phänomen
}

\author{
Fake News as a socio-linguistic phenomenon \\ Anna DASZKIEWICZ ${ }^{1}$ \\ Ateneum-Szkoła Wyższa w Gdańsku
}

\begin{abstract}
This paper addresses the socio-linguistic phenomenon of fake news. The emphasis is put on the premises, impact, perception, reception, and further functioning of purposefully falsified information. The text provides both a theoretical basis as well as practical fields of its application.

Keywords: Fake News, falsified information, purposefully distorted messages, social media platforms, the sleeper- or illusory-truth-effect, influence and further functioning of falsified news

\section{Zusammenfassung}

Der vorliegende Beitrag wendet sich dem soziolinguistischen Phänomen „Fake News“ zu. Hierbei liegt der besondere Fokus auf Anliegen, Aussagekraft, Wahrnehmung und Nachwirkung von gezielt gefälschten Botschaften. Der Beitrag liefert sowohl theoretische Grundlagen, als auch praktische Anwendungsfelder dazu.

Schlüsselwörter: Fake News, Falschnachrichten, gezielt gefälschte Botschaften, Social-MediaPlattformen, der Sleeper- oder der Illusory-Truth-Effekt, die Auswirkung und Nachwirkung von Falschnachrichten
\end{abstract}

1 (D) https://orcid.org/0000-0003-3745-8528. 


\section{Definitorischer Teil}

Im vorliegenden Beitrag wende ich mich dem Phänomen der geschriebenen Sprache, und zwar bewusst gefälschten Pressemittleilungen, den sogenannten Fake News² zu. Der Begriff, der spätestens seit dem Wahlsieg von Donald Trump (2016) eine Runde macht und sich vorwiegend in politischen und sozial-kulturellen Kontexten wie ein Lauffeuer verbreitet, wird aus dem Englischen abgeleitet und steht für "falsche“ oder "gefälschte“ Nachrichten. Dabei wir der besagte Begriff derzeit immer umfangreicher und umfasst demnach „alle möglichen Formen von als problematisch eingestuften, medial verbreiteten Inhalten“ (Müller und Denner, 2019, S. 6). Unter den als „problematisch“ wahrgenommenen "Inhalten“ kann es sich sowohl um absichtlich gefälschte, als auch ungenaue, versehentlich oder nicht gezielt verbreitete, oder nicht akkurate Informationen handeln. Im engen Zusammenhang damit sind allerdings auch Beiträge kaum wegzudenken, die „reißerische Schlagzeilen und/oder eine hasserfüllte und zweifelhafte Rhetorik“ (Ibidem) enthalten. Zudem bezieht sich die Begriffsverwendung auch auf wissenschaftliche Studien oder historische Informationen. Wie daraus ersichtlich, mangelt dem derzeitigen Gebrauch des Begriffs in dem öffentlichen Diskurs an definitorischer Schärfe. Dieser Mangel wird nur dadurch vermehrt,

dass sich politische Akteure, allen voran der aktuelle Präsident der Vereinigten Staaten von Amerika, erfolgreich an einer Umdeutung des Begriffes erprobt haben. Sie verwenden ihn als Bestandteil einer populistischen Rhetorik, um etablierte journalistische Leitmedien der systematischen und absichtlichen fehlerhaften Berichterstattung zu bezichtigen. (Schulz, Wirth und Müller, 2018)

Trotz besagter Vielzahl an Begriffsauslegungen steht eines fest: Fake News werden im Generellen über das Internet (insbesondere über Facebook und Twitter) verbreitet, geteilt und im Nachhinein viel tausendfach und

\footnotetext{
2 „Der Begriff ,Fake News` ist der Anglizismus des Jahres 2016. Neben der ,überwältigenden und anhaltenden öffentlichen Präsenz' fülle der Begriff eine Lücke im deutschen Wortschatz, teilte die Jury um den Sprachwissenschaftler Anatol Stefanowitsch von der Freien Universität Berlin zur Begründung mit. Die Bezeichnung ,Fake News‘ wurde nach Angaben der Wissenschaftler im Englischen etwa seit dem Ende des 19. Jahrhunderts für bewusste Falschmeldungen in Zeitungen verwendet. ,Der Durchbruch in den allgemeinen Sprachgebrauch erfolgte erst ab November 2016 im Zusammenhang mit einer Bedeutungsverschiebung hin $\mathrm{zu}$ politisch motivierten Falschmeldungen, die angeblich dem Kandidaten Donald Trump den Sieg im Präsidentschaftswahlkampf in den USA bescherten', erklärte die Jury“ (https:/www.dw.com/ de/fake-news-ist-anglizismus-des-jahres/a-37386704; Zugriff auf den 23.03.2020).
} 
mitunter sogar millionenfach weitergeleitet. Auf diese Art und Weise werden Falschmeldungen jedoch nur ins Rollen gebracht: Mehrfach generierte Mausklicks sorgen für besondere Aufmerksamkeit und gewährleisten hohe Werbeeinnahmen.

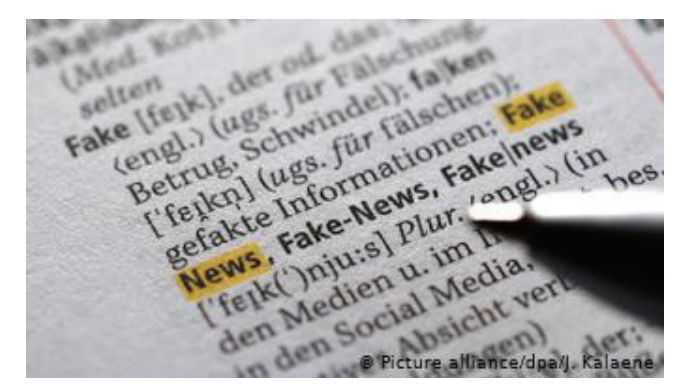

Abb. 1. Seit 2017 gibt es "Fake News" als Begriff im Duden

(Das Bild: https://www.dw.com/de/papst-franziskus-warnt-vor-fake-news-und-mahntjournalisten-zu-wachsamkeit/a-42286371)

Nun aber verbirgt sich dahinter noch eine andere Idee: Die gezielte Irreführung und Beeinflussung der öffentlichen Meinung, wobei zusätzlich das Kriterium erfüllt werden muss, sich auf aktuelle Geschehnisse zu beziehen und damit nachrichtlichen Charakter zu bewahren (Zimmermann und Kohring, 2018). Dabei ist die bereits erwähnte Desinformation der öffentlichen Meinung gar nicht neu:

Als Bestandteil der Berichterstattung journalistischer Massenmedien sind Falschmeldungen unter den Namen ,Zeitungsente' (Hollstein, 1991) oder ,Tatarenmeldung' (Walther, 2016) schon seit dem 19. Jahrhundert bekannt. Allerdings kann unterstellt werden, dass klassische Falschmeldungen von professionellen Journalisten in erster Linie unabsichtlich verbreitet wurden bzw. werden und im Normalfall berichtigt werden, sobald ihre Unwahrheit bekannt wird. Sie fallen damit nicht unter die Kategorie Desinformation. (Müller und Denner, 2019, S.7)

Als treffende Beispiele hierfür dienen die 1986 von der Sowjetunion zunächst verbreitete Mitteilung über die mutmaßliche Harmlosigkeit des Reaktorunglückes von Tschernobyl oder aber auch die Aussage über die vermeintliche Ermordung von Babys durch irakische Soldaten im Golfkrieg 1991, einhergehend mit in demselben Zusammenhang gefälschten Unterlagen über irakische Massenvernichtungswaffen, die dem UN-Sicherheitsrat 
zur Begründung des amerikanischen Angriffs vorgelegt, für die weltweite Empörung sorgten.

Wie aus den oben veranschaulichten Beispielen ersichtlich, handelt es sich im Fall von Falschmeldungen insbesondere um eine absichtliche und flächendeckende Propaganda aus rechtspopulistischen Kreisen, der nicht erhabene, sondern vielmehr die öffentliche Meinung polarisierende und vernichtende Ziele innewohnen. Dabei werden meist heikle und moralisch aufgeladene Fragestellungen wie etwa Migranten und Flüchtlinge, Kinder und Missbrauch oder Krieg und Frieden aufgegriffen und in Form einer durchaus schlichten und einseitigen Berichterstattung veröffentlicht. Meist kommen Fake News ohne großen Text aus; sie treten also gewöhnlich in Form nur eines Bildes mit dicker Schlagzeile auf. Übrigens werden mittels zugänglicher, leicht erkennbarer Aussagen einfache Wahrheiten versprochen, wobei die Welt komplex ist und es keine einfachen Erklärungen oder Lösungswege für komplizierte Probleme gibt. Sowohl die Auswahl der aus der nahen Umgebung stammenden Themenfelder sowie die der stilistischen Mittel hat bei Rezipienten eine sofortige Reaktion zu wecken und im Endeffekt dazu zu führen, dass Falschinformationen in sozialen Netzwerken verarbeitet und weiter verbreitet werden. Vor diesem Hintergrund scheint es eine große Rolle zu spielen, ob Fake News in Kreisen von Nutzern mit ähnlichen Einstellungen und Weltanschauungen ausgetauscht und geteilt werden. Sollte dies der Fall sein, haben Fake News die Chance, ein breiteres Publikum zu erreichen, sprich zu erwischen:

Je homogener der Kreis an Nutzern ist, die eine Nachricht teilen, umso größer ist die Wahrscheinlichkeit, dass sie immer weiter geteilt wird und somit ein großes Publikum erreicht. Besonders eindeutige ,Fake News', die eine deutliche politische Botschaft enthalten, haben also beste Chancen viel Aufmerksamkeit zu erregen. (Ibidem, S. 8)

Wie bereits zuvor angemerkt, kommen Fake News ohne großen Text aus. Sie verkörpern vorwiegend Nachrichtenteaser, d.h. Schlagzeilen und wenige Zeilen umfassende Zusammenfassungen einer Meldung. Diese Teaser lassen sich schnell erfassen und mit wenig kognitivem Aufwand verarbeiten (Costera Meijer und Groot Kormelink, 2015; Schäfer, Sülflow und Müller, 2017). Daher soll davon ausgegangen werden, dass im Fall von Fake News eher Nachrichtenteaser als sich unter ihnen verbergenden vollständigen Nachrichten wahrgenommen werden. Zusammengenommen spricht dies dafür, dass Fake News auf Social-Media-Plattformen selbst bei Nutzern, die sich 
eigentlich der Tatsache bewusst sind, dass Informationen auf diesen Plattformen nicht zwangsläufig hohen Qualitätsstandards entsprechen und möglicherweise unwahr sein könnten, weder hinterfragt noch kritisch angesehen werden. Der vermutete Mechanismus dahinter besteht darin, dass Menschen im Generellen dazu tendieren, Informationen und darunter auch Nachrichteninhalte so wahrzunehmen, dass sie im Einklang mit ihren bereits vorhandenen Überzeugungen und Vorstellungen stehen. Dies beginnt schon bei der Auswahl der Meldungen, die gelesen werden (Selective Exposure) und setzt sich bei der Auslegung und Deutung (Selective Perception) sowie der späteren Erinnerung an die wahrgenommenen Inhalte (Selective Retention) fort (Frey, 1986; Zillmann und Bryant, 1985). Vor diesem Hintergrund trage die Nachrichtenwahrnehmung über Social-MediaPlattformen nicht selten zu einer sogenannten Filter Bubble bei (Pariser, 2011). Demzufolge wendet man sich bevorzugt den Informationen $\mathrm{zu}$, die die vorgefestigte Meinung bzw. das bestehende Weltbild verkörpern und belegen. So verstärkt sich der Filter-Bubble-Effekt, der seine Wurzeln in den kognitiven Strukturen des einzelnen Rezipienten hat.

Nun aber mag bei einer derartigen Verarbeitung neu auftretender Informationen $\mathrm{zu}$ einer kognitiven Abkürzung kommen, was wiederum auf einen heuristischen Modus der Informationsverarbeitung hindeute (D'Alessio und Allen, 2002). Denn es wird in der Forschung auf zwei grundlegende Modi verwiesen, wie Menschen mit neuen Inhalten umgehen (Chaiken, Liberman und Eagly, 1989). Demzufolge können Inhalte entweder heuristisch oder systematisch aufgefasst und weiterverarbeitet werden. Der heuristische Modus setzt einen geringen kognitiven Aufwand bei der Nachrichtenrezeption voraus. Beim systematischen Modus hingegen denken Rezipienten gründlich über alle Aspekte nach und wägen die aus einer Meldung zu ziehenden Schlüssen gut ab. Letzteres trifft aber nur dann zu,

wenn eine hohe persönliche Motivation vorliegt, die den Aufwand der systematischen Verarbeitung gerechtfertigt erscheinen lässt. Dies ist z. B. der Fall, wenn ein großes Interesse am Thema einer Nachricht besteht oder wenn eine persönliche Betroffenheit gegeben ist. Insgesamt muss daher davon ausgegangen werden, dass die meisten Menschen Nachrichteninhalte eher heuristisch verarbeiten (Brosius, 1995; Graber, 1988, zitiert nach Müller \& Denner 2019, S. 14).

Jüngere Studien lassen das oben skizierte Schema der Nachrichtenrezeption und -Auslegung nur noch bestätigen: Mangelndes 
Nachdenkvermögen erhöht erheblich die Gefahr, Falschnachrichten für wahr zu halten (Pennycook \& Rand, 2018). Die Gefahr, eine gefälschte Nachricht als glaubwürdig anzusehen, sinkt, wie bereits vorstehend beleuchtet, mit steigenden kognitiven Ressourcen, die ein potenzieller Rezipient für die Verarbeitung einer jeweiligen Nachricht mitbringen kann. Werden die kognitiven Ressourcen allerdings kaum aktiviert, werden gefälschte Mitteilungen zu einem potentiell gefährlichen Mittel der politischen Kommunikation. Denn im Endeffekt bedeutet der heuristische Modus: „Wenn der Inhalt einer ,Fake News' zu dem Weltbild passt, das ein Rezipient bereits mitbringt, ist die Wahrscheinlichkeit hoch, dass die entsprechende Meldung geglaubt und nicht hinterfragt wird“ (Müller und Denner 2019, S. 15).

Aber auch wer der Quelle einer gefälschten Meldung zunächst kritisch gegenübersteht, kann nicht völlig immun dagegen sein, von deren Botschaft beeinflusst zu werden. Es handelt sich hier um einen Sleeper-Effect, der in den letzten Jahrzehnten durch eine Reihe von Studien wiederholt nachgewiesen wurde (Kumkale und Albarracín, 2004). Demnach vergessen Rezipienten nach einiger Zeit die Quelle, aus der sie eine Meldung ursprünglich einmal hatten und damit auch, ob sie einst die Quelle als vertrauenswürdig angesehen haben. Die Information an sich verbleibt allerdings in ihrer Erinnerung. Im Zusammenhang damit sei hinzugefügt: „Allerdings funktioniert dies vor allem dann besonders gut, wenn sie [die Nachricht] zum existierenden Vorwissen der jeweiligen Person passt:

Das Auftreten eines solchen Sleeper-Effects ist also dann besonders wahrscheinlich, wenn Rezipienten mit ,Fake News' konfrontiert sind, die inhaltlich ihren Erwartungen und ihrem Weltbild entsprechen. Dann kann es passieren, dass sie der Quelle im ersten Moment misstrauen, dies jedoch mit der Zeit vergessen und sich letztlich nur noch an die zunächst für unwahr gehaltene, aber später doch geglaubte Botschaft erinnern können. Dass der Sleeper-Effect grundsätzlich bei Nachrichtenposts auf Sozial Media greift, konnte bereits nachgewiesen werden. (Heinbach, Ziegele und Quiring 2018, zitiert nach Müller \& Denner, 2019, S.16)

Während sich der Sleeper Effect im überwiegen Maße auf diejenigen Rezipienten bezieht, die einem Fake lediglich aufgrund der Quelle misstrauen, diesen inhaltlich aber für wahrscheinlich halten, gibt es noch einen weiteren Effekt von Fake News, und zwar den Illusory Truth Effect, der selbst bei denjenigen, die fundamentale inhaltliche Zweifel haben, eintreten kann: 
Eine Reihe von Studien hat gezeigt, dass Aussagen, denen Rezipienten wiederholt und am besten aus verschiedenen Quellen begegnen, irgendwann geglaubt werden, selbst wenn sie anfänglich für falsch gehalten werden (,Illusory Truth Effect', Dechêne, Stahl, Hansen, \& Wänke 2010). [...] Verallgemeinert bedeutet dies: Wer eine ,Fake News' wiederholt zu sehen bekommt, beginnt vermutlich irgendwann, ihr zu glauben, weil sie ihm bekannt vorkommt. (Ibidem)

Bedauerlicherweise erzeugen meist sämtliche Versuche, Rezipienten über das Wirkpotenzial von Fake News aufzuklären und sie vor einer unkritischen Fake News-Rezeption zu warnen, nur eine Reaktanz bei ihnen. Bei wenig kritischen Sozial-Media-Nutzern können Warnhinweise oft kaum zur Korrektur des zuvor getroffenen Urteils (de Keersmaecker und Roets, 2017) verhelfen, sondern eher Wut und Trotz hervorrufen. Kurzum wird dadurch an den Inhalt der Fälschungen erinnert, statt deren Verbleib wirksam bekämpft.

\section{Empirischer Teil}

Es sei an dieser Stelle in die meist bekannten Fake News, die in den letzten Jahren die öffentliche Meinung weltweit berührt haben, eingeweiht und dafür überhaupt sensibilisiert. Hierbei werden die zur Analyse gewählten Falschnachrichten samt der Angabe deren Quelle und Richtigstellung veranschaulicht und unten noch ausführlich besprochen.

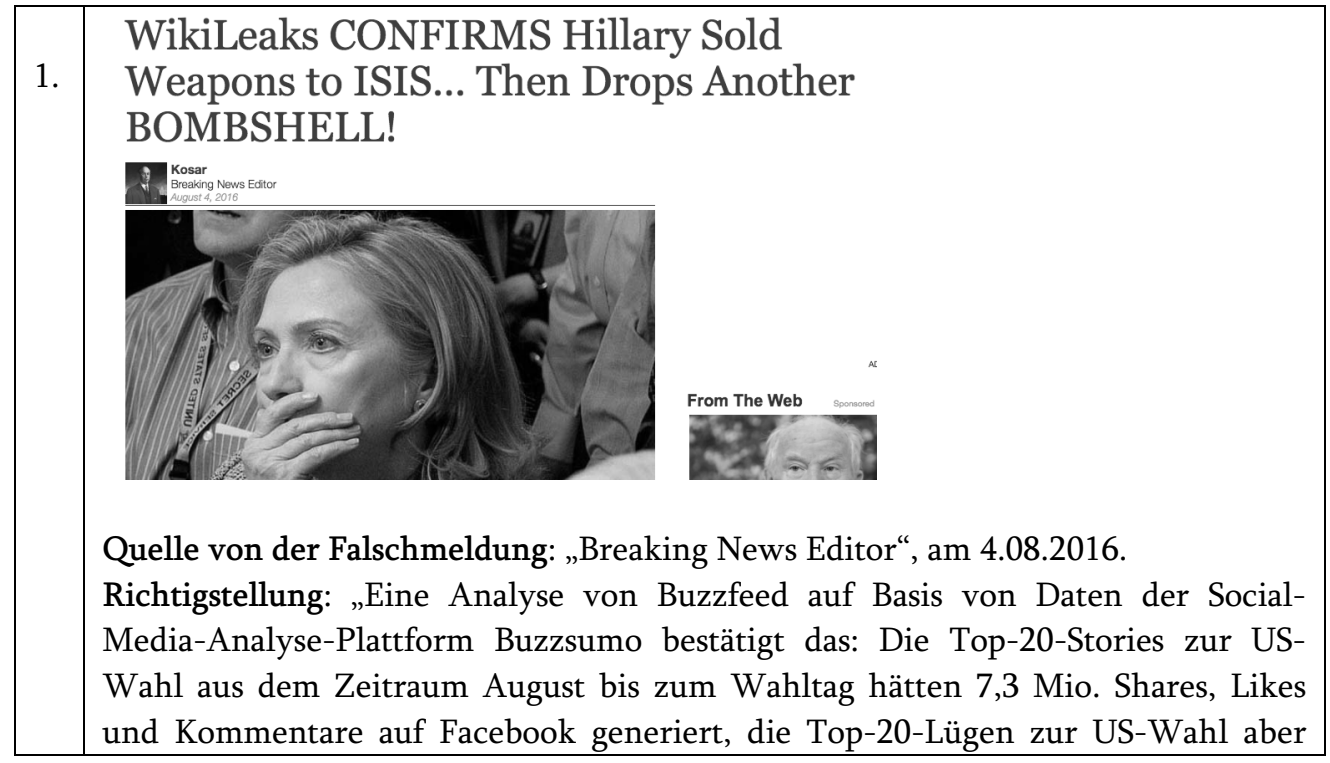




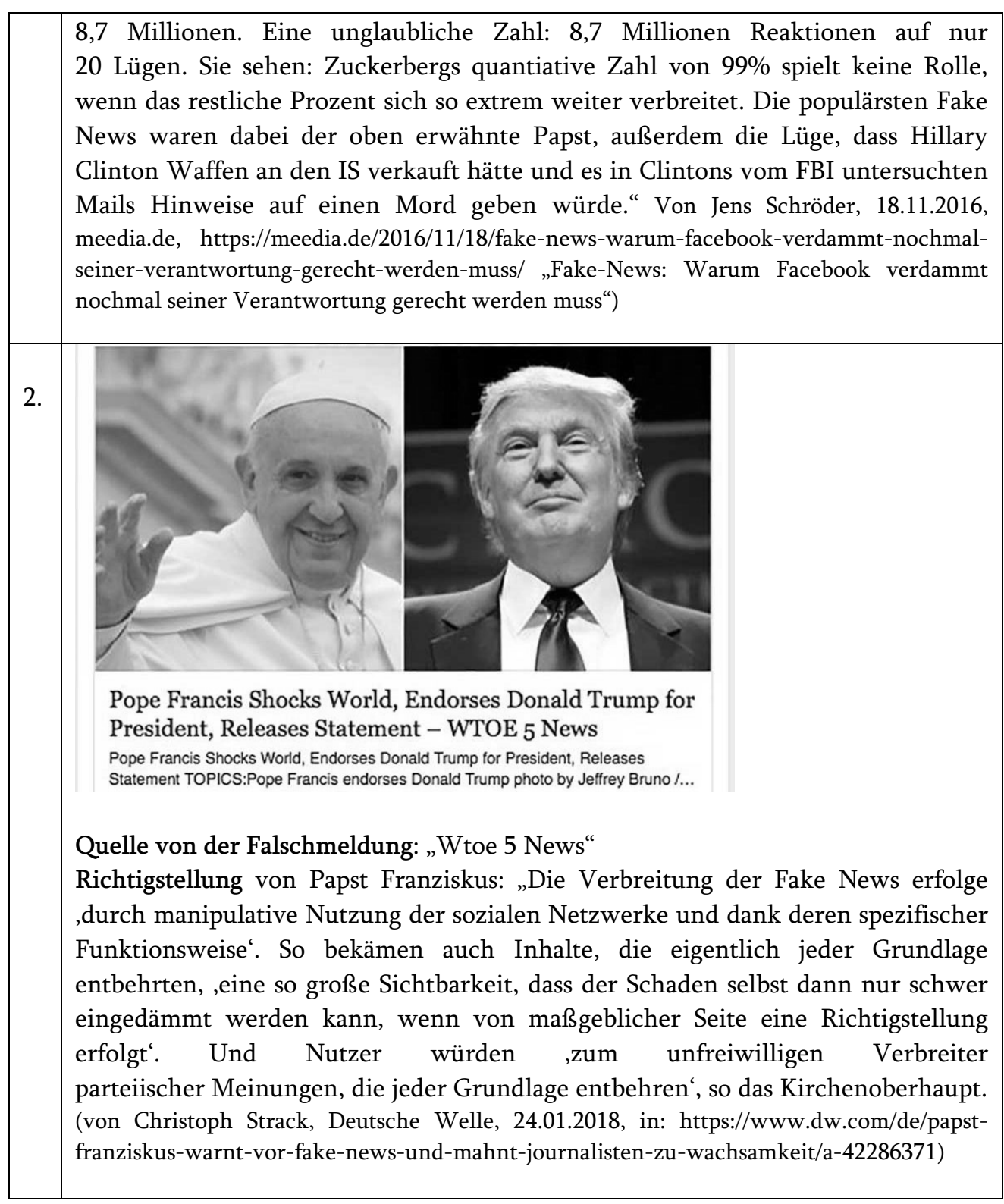




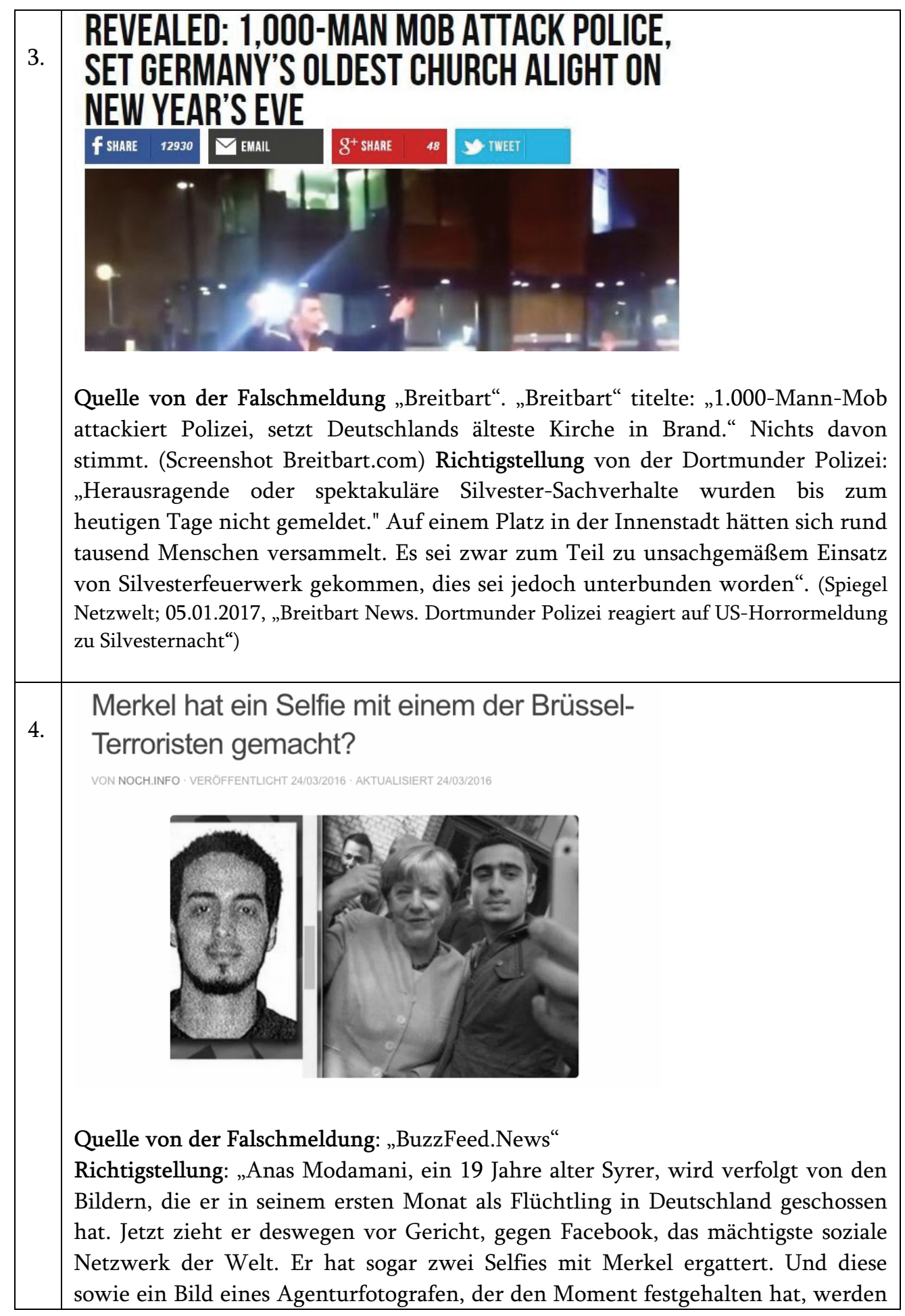




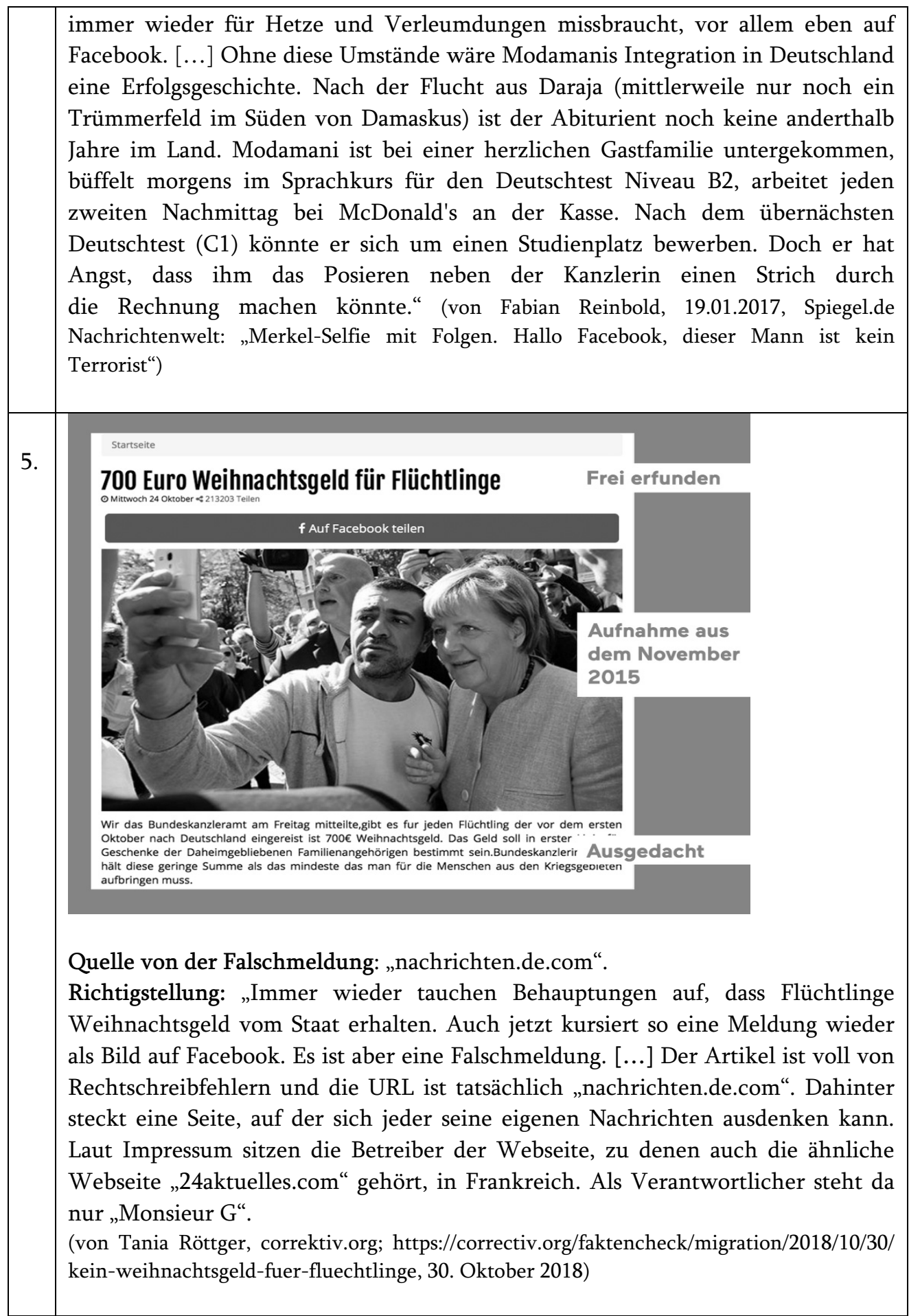


Wie aus den oben veranschaulichten Beispielen hervorgeht, werden zur Zielscheibe der Fake News-Attacken vor allem gesellschaftlich anerkannte, prominente Figuren mit einem erhabenen Ruf (wie beispielsweise die Bundeskanzlerin Angela Merkel oder der Bischof von Rom und zugleich das Oberhaupt der römisch-katholischen Kirche, der Papst Franziskus). Diese werden ungewollt und meistens völlig unbewusst in ein durchaus unanständiges Spiel verwickelt, in dem es nur darum geht, die Aufmerksamkeit auf subjektiv ausgewählte und angestrebte Zwecke zu lenken und somit den größtmöglichen Gewinn zu erzielen. Demnach fungieren Akteure der öffentlichen Szene sowie des öffentlichen Vertrauens als Marionetten oder Schachfiguren, die man je nach dem zu erreichenden Ziel hin- und herschiebt, sprich ausnutzt. Dabei wird wenig um deren Wohlbefinden und Ansehen geschert. Ganz im Gegenteil: Nur noch auf den eigenen Vorteil bedacht, bedienen sich Urheber von Fake News gezielt der einflussreichen Figuren des öffentlichen Lebens, hinterfragen deren Handlungsfähigkeit als Oberhaupt von Staat und Kirche, setzen deren Verdienste und Fähigkeiten rücksichtslos ab und beschädigen im Endeffekt enorm, wenn überhaupt nicht unwiderruflich, ihren Ruf. Allesamt Maßnahmen, die die öffentliche Meinung nur $\mathrm{zu}$ verunsichern (auf diesem Weg werden alte Ressentiments gegenüber Andersstämmigen oder ungewollten Kandidaten geweckt) und das Vertrauen in die Regierung und das politische System in dem jeweiligen Land auszuhöhlen haben.

Andererseits machen die oben angegebenen Fake News-Repräsentanten deutlich, dass sich der beabsichtigte Effekt auch mit einem negativ auffallenden, nachlässigen Schreibstil, einer saloppen bis derben Ausdrucksweise erzielen lässt. Es wimmelt in Falschnachrichten nicht selten von Rechtschreib-, Grammatik- und Interpunktionsfehlern, die entweder aus Unwissenheit oder Nachlässigkeit herkommen und gering ausgebildete Schreibkompetenzen bei deren Verfassern aufweisen, oder vielleicht ganz umgekehrt, das Ziel verfolgen, als möglichst simpel und schrill rüberzukommen. Des Weiteren liegt hier der Fokus auf einem appellativen Ton mancher Interpunktionsmittel wie etwa der Ausrufe- bzw. Fragezeichen, die zumal vermehrt eingesetzt und deutlich hervorgehoben, zu einem besseren Verleumdungs-, Unterstellungs- und Verhöhnungseffekt verhelfen oder dazu einen wichtigen Beitrag leisten. Dies wiederum besagt, dass das Wirkpotenzial von Fake News überwiegend auf diejenigen zutrifft, die nicht für Stringenz und Qualität einer jeweiligen Meldung, sondern vielmehr für populistische 
Parolen empfänglich sind und Fake News grundsätzlich heuristisch verarbeiten.

Hierbei lassen sich wenig kritische, nicht viel fordernde Leser reibungslos durch nachlässige Informationsquellen bedienen. Sämtliche im vorliegenden Beitrag angeführten Falschnachrichten liefern einen wichtigen Beweis für deren zweifelhaften und nicht durch Fakten gesicherten Ursprung. Darauf verweisen Journalisten, die ihre Aufgabe darin sehen, Fake News als Lügen zu entlarven und somit die ihrem Beruf innewohnende Glaubwürdigkeit wiederherzustellen und zu festigen. Im Zusammenhang damit seien an dieser Stelle einige journalistische Projekte wie Lie Detectors, Correktiv / Correktiv.org oder Hoaxmap / Hoaxmap.org erwähnt, die dazu verhelfen, eine absichtlich ins Netz gestellte Lüge als eine nicht perfekte journalistische Berichterstattung aufzufassen und im Übrigen auch vermitteln, „dass die Mitte, das Ungenaue, auch sehr interessant sein kann, ohne dass man sich immer sofort auf gegensätzliche Pole zurückziehen muss. Letztlich geht es um Demokratie - und gegen Radikalisierung" (Fokken, 2019), so die Gründerin und Leiterin der Organisation Lie Detectors, die Journalistin Juliane von Reppert-Bismarck.

\section{Schlussbemerkungen}

Im vorliegenden Beitrag wird dem Phänomen Fake News nachgegangen. In den gesellschaftlichen Fokus gerückt (Falschmeldungen sind in Zeiten netzgetriebener Nachrichtenkommunikation extrem unübersichtlich), erweisen sich mit Absicht gefälschte und medial verbreitete Inhalte als ein dankbares und eindringliches Untersuchungsobjekt, das allerdings noch der näheren Beleuchtung bedarf. Nichtsdestotrotz liefert der vorliegende Beitrag, zumal theoretisch gesichert und empirisch gestützt, genügend Beweise für deren unabdingbares und schädliches Wirkpotenzial (erwähnt seien an dieser Stelle unter anderem der Sleeper- oder der Illusory-Truth-Effect. Doch Fake News können auch Rezipienten beeinflussen, die für ihre Aussagen prinzipiell nicht anfällig sind) und lässt sie überhaupt erst als Geflecht aus Gerüchten erleben und betrachten, die leicht und weltweit abrufbar veröffentlicht, meist um politische Gegner oder Andersstämmige (Flüchtlinge) gerankt, Stimmung gegen sie machen, sprich Angst und Hass schüren. Des Weiteren verdeutlicht der Beitrag, dass die Rezeption von Fake News im überwiegenden Maße über Sozial-Media-Plattformen erfolgt, und zwar in den meisten Fällen in einem heuristischen Verarbeitungsmodus. Es wird hier außerdem auf die am häufigsten im Internet kursierten Fake News 
zurückgegangen, für deren schriftliche Form und Aussagekraft sensibilisiert sowie für deren Richtigstellung gesorgt. Alles in allem sollte der vorliegende Beitrag auf die Problematik der netzgetriebenen Nachrichtekommunikation Aufmerksamkeit lenken und zur weiteren Recherche auf diesem Gebiet anregen.

\section{Bibliografie}

Brosius, H.-B. (1995). Alltagsrationalität in der Nachrichtenrezeption - Ein Modell zur Wahrnehmung und Verarbeitung von Nachrichteninhalten. Wiesbaden: Westdeutscher Verlag.

Chaiken, S., Liberman, A. \& Eagly, A. H. (1989). Heuristic and systematic information processing within and beyond the persuasion context. In J. S. Uleman \& J. A. Bargh (Hrsg.), Unintended Thought (S. 212-251). New York: Guilford Press.

Costera Meijer, I., \& Groot Kormelink, T. (2015). Checking, sharing, clicking and linking. Changing patterns of news use between 2004 and 2014. Digital Journalism, 3(5), 664-679.

D'Alessio, D. \& Allen, M. (2002). The selective exposure hypothesis and media choice processes. In R. W. Preiss, B. M. Gayle, N. Burrell, M. Allen, \& J. Bryant (Hrsg.), Mass Media Effects Research: Ad-vances through Meta-Analyses (S. 103-118). New York: Routledge.

Dechêne, A., Stahl, C. Hansen, J., \& Wänke, M. (2010). The truth about the truth: A meta-analytic review of the truth effect. Personality and Social Psychology Review, 14(2), 238-257.

Fokken, S. (15.10.2019). Workshops gegen Desinformation. "In jeder Klasse fällt mindestens ein Kind auf Fake News herein". Zugriff auf den 23.03.2020 https://www.spiegel.de/lebenundlernen/schule/jugendlicher-populismus-wiedie-lie-detectors-fake-news-enttarnen-a-1291695.html.

Frey, D. (1986). Recent research on selective exposure to information. In L. Berkowitz (Hrsg.), Advances in Experimental Social Psychology (Vol. 19, S. 41-80). New York: Academic Press.

Graber, D. A. (1988). Processing the News: How People Tame the Information Tide. New York: Longman.

Heinbach, D., Ziegele, M. \& Quiring, O. (2018). Sleeper effect from below: Long-term effects of source credibility and user comments on the persuasiveness of news articles. New Media \& Society, Vol. 20/12, 4765-4786. https://doi.org/10.1177/1461444818784472, Zugriff auf den 23.03.2020. 
Hollstein, H. (1991). Zeitungsenten. Kleine Geschichte der Falschmeldung. Heitere und ernste Spielarten vom Aprilscherz bis zur Desinformation. Stuttgart: Bertelsen.

de Keersmaecker, J. \& Roets, A. (2017). 'Fake news': Incorrect, but hard to correct. The role of cognitive ability on the impact of false information on social impressions. Intelligence, 65, 107-110.

Kumkale, G. T. \& Albarracín, D. (2004). The sleeper effect in persuasion: A metaanalytic review. Psychological Bulletin,130(1), 143-172.

Müller, P. \& Denner, N. (2019): Was tun gegen Fake News? 2. Aufl., Berlin, FriedrichNaumann-Stiftung.

Pariser, E. (2011). The Filter Bubble. London: Penguin.

Pennycook, G. \& Rand, D. G. (2018). Lazy, not biased: Susceptibility to partisan fake news is better ex-plained by lack of reasoning than by motivated reasoning. Cognition. https://doi.org/10.1016/j.cognition.

Reinbold, F. (19.01.2017). Spiegel.de Nachrichtenwelt: „Merkel-Selfie mit Folgen. Hallo Facebook, dieser Mann ist kein Terrorist“, Zugriff auf den 23.03.2020 https://www.spiegel.de/netzwelt/web/angela-merkel-ein-selfie-mit-folgenhallo-facebook-dieser-mann-ist-kein-terrorist-a-1130400.html.

Röttger, T. (30.10.2018) correktiv.org. Zugriff auf den 23.03.2020, https://correctiv.org/ faktencheck/migration/2018/10/30/kein-weihnachtsgeld-fuer-fluechtlinge,

Schäfer, S., Sülflow, M. \& Müller, P. (2017). The special taste of snack news: An application of niche theory to understand the appeal of Facebook as a source for political news. First Monday, 22(4). https://doi.org/10.5210/fm.v22i4.7431

Schröder, J. (18.11.2016). „Fake-News: Warum Facebook verdammt nochmal seiner Verantwortung gerecht werden muss“. meedia.de. Zugriff auf den 23.03.2020 https://meedia.de/2016/11/18/fake-news-warum-facebook-verdammt-nochmalseiner-verantwortung-gerecht-werden-muss

Schulz, A., Wirth W. \& Müller, P. (2018). We are the people and you are fake news: A social identity approach to populist citizens' false consensus and hostile media perceptions. Communication Research. Vol. 47/2, 201-226. DOI: $10.1177 / 0093650218794854$

Spiegel Netzwelt (05.01.2017). „Breitbart News“. Dortmunder Polizei reagiert auf USHorrormeldung zu Silvesternacht“, Zugriff auf den 23.03.2020 https://www.spiegel.de/netzwelt/web/dortmund-polizei-reagiert-auf-breitbarthorrormeldung-zu-silvester-a-1128787.html,

Strack, Ch. (24.01.2018). Papst Franziskus warnt vor Fake News und mahnt Journalisten zu Wachsamkeit. Deutsche Welle. Zugriff auf den 23.03.2020 https://www.dw.com/de/papst-franziskus-warnt-vor-fake-news-und-mahntjournalisten-zu-wachsamkeit/a-42286371

Walther, R. (2016, 30. November). Fotografie: Wo Bilder zu Waffen werden. Die Zeit. Zugriff auf den 23.03.2020 https://www.zeit.de/2006/49/P-Daniel 
Zimmermann, F. \& Kohring, M. (2018). „Fake News“ als aktuelle Desinformation. Systematische Bestimmung eines heterogenen Begriffs. Medien \& Kommunikationswissenschaft, 66(4), 526-541. 
